\title{
Diet and risk of breast, endometrial and ovarian cancer: UK Women's Cohort Study
}

\author{
Yashvee Dunneram $^{1 *}$, Darren C. Greenwood ${ }^{2}$ and Janet E. Cade ${ }^{1}$ \\ ${ }^{1}$ Nutritional Epidemiology Group, School of Food Science E Nutrition, University of Leeds, Leeds LS2 9JT, UK \\ ${ }^{2}$ Division of Epidemiology and Biostatistics, University of Leeds, Leeds LS2 9NL, UK \\ (Submitted 29 June 2018 - Final revision received 30 October 2018 - Accepted 27 November 2018; First published online 11 December 2018)
}

\section{Abstract}

This study aimed to investigate the association between diet and the risk of breast, endometrial and ovarian cancer in the UK Women's Cohort Study. A total of 35372 women aged 35-69 years were enrolled between 1995 and 1998 and completed a validated 217-item FFQ. The individual foods were collapsed into sixty-four main food groups and compared using Cox proportional models, adjusting for potential confounders. Hazard ratio (HR) estimates are presented per portion increase in food items. After approximately 18 years of follow-up, there were 1822, 294 and 285 cases of breast, endometrial and ovarian cancer, respectively. A high consumption of processed meat and total meat was associated with an increased risk of breast and endometrial cancer. High intake of tomatoes (HR 0.87, 99\% CI 0.75, 1.00) and dried fruits (HR 0.60, 99\% CI 0.37, 0.97) was associated with a reduced risk of breast and endometrial cancer, respectively. Mushroom intake was associated with a higher risk of ovarian cancer (HR 1.57, $99 \%$ CI 1.09, 2.26). Subgroup analysis by pre- or postmenopausal cancer further demonstrated an association between processed meat intake and both postmenopausal breast cancer and endometrial cancer. Intake of dried fruits was associated with a reduced risk of postmenopausal endometrial cancer (HR 0.55, $99 \%$ CI 0.31, 0.98). Our findings suggest that while some foods may trigger the risk of these cancers, some foods may also be protective; supporting the call for further randomised controlled trials of dietary interventions to reduce the risk of cancer among pre- and postmenopausal women.

Key words: Diet: Food groups: Breast cancer: Endometrial cancer: Ovarian cancer: Premenopausal women: Postmenopausal women

In the UK, breast cancer is the most commonly diagnosed cancer among women accounting for almost one-third of all female cancers. Endometrial and ovarian cancers are the next most frequently diagnosed hormone-related cancers among British women ${ }^{(1)}$. These cancers are all age dependent and commonly diagnosed postmenopausally ${ }^{(2)}$. The mechanisms involved in the pathogenesis of these cancers are not completely elucidated. Reproductive and hormonal risk factors such as an early age at menarche, late age at menopause, lack of oral contraceptive use, lack of tubal ligation, postmenopausal hormone therapy, nulliparity, all contribute to the lifetime oestrogen exposure $^{(3,4)}$ as well as a family history have been consistently associated with these reproductive cancers ${ }^{(5)}$. Moreover, smoking has also been associated with an increased risk of breast and ovarian cancers while it reduces the risk of endometrial cancer ${ }^{(6,7)}$. In addition, evidence from observational studies has indicated that obesity-related metabolic disorders such as diabetes and the metabolic syndrome can be linked to the aetiology of these cancers ${ }^{(8)}$. These metabolic disorders are partly outcomes of poor dietary quality ${ }^{(9)}$.
In addition to being one of the triggering factors in the development of obesity, diet also potentially influences the endogenous hormonal milieu, thereby increasing the risk of these hormone-related cancers ${ }^{(10)}$. As demonstrated in previous studies, dietary changes have been linked to changes in menstrual cycle length, circulating sex hormone-binding globulin levels and also oestradiol levels ${ }^{(11-14)}$. Even though studies have shown that diet may be related to the risk of breast, endometrial and ovarian cancer, the specific dietary components involved in the aetiology of these cancers remain unclear. For instance, according to the recent World Cancer Research Fund/American Institute for Cancer Research report ${ }^{(15)}$, there was strong evidence that alcohol consumption increases both the risk of pre- and postmenopausal breast cancers. In addition, there was suggestive evidence demonstrating that a high consumption of non-starchy vegetables, foods sources of carotenoids, dairy products and Ca-rich diets were associated with a decreased risk of breast cancer. On the other hand, the link between other foods and risk of breast cancer remains limited and inconclusive. Likewise, the relationship between diet and endometrial

Abbreviations: HR, hazard ratio; HRT, hormone replacement therapy; ICD, International Classification of Diseases; UKWCS, UK Women's Cohort Study.

* Corresponding author: Y. Dunneram, email fsyd@leeds.ac.uk 
as well as ovarian cancer was sparse and conflicting. Therefore, using data from the UK Women's Cohort Study (UKWCS), this study aims to investigate the associations between food intake and the risk of breast, endometrial and ovarian cancer.

The aetiology of these cancers also differs by whether the cancer is pre- or postmenopausal. While evidence suggests a link between endogenous oestrogens and risk of these cancers among postmenopausal women, there is only weak evidence supporting this relationship among premenopausal women ${ }^{(16,17)}$. In addition, the menstrual cycle variations in circulating sex hormone levels make deciphering the aetiology behind premenopausal breast, endometrial and ovarian cancer risk a challenge ${ }^{(18)}$. This study thus also seeks to look into the relationship between diet and risk of the hormone-dependent cancers by menopausal status.

\section{Methods}

\section{Study design, study population and ethical approval}

At baseline, the UKWCS involved 35372 women across England, Wales and Scotland who responded to a postal questionnaire between 1995 and 1998. The recruitment process has been detailed elsewhere ${ }^{(19)}$. Recruited women were aged between 35 and 69 years. Dietary data, lifestyle as well as health-related data were collected at baseline. Approximately 4 years later, further diet, lifestyle and health-related data were collected between the years 1999 and 2002 (40.1\% response), which formed the follow-up cohort. Reproductive history including menopausal status was also collected at study baseline and follow-up. At its initiation in 1993, ethical approval was obtained from 174 local research ethics committees (Research Ethics Committee reference number: 15/YH/0027).

\section{Dietary assessment}

A detailed validated ${ }^{(20)} 217$-food item FFQ was used to assess the dietary intake of the participants over a period of 12 months. Daily intake of each food item $(\mathrm{g} / \mathrm{d})$ was determined using the frequency categories to estimate the portion size. Using a standard portion size, these were then converted into weights. According to the recent World Cancer Research Fund report, one of the identified critical areas of research included better characterisation of diet ${ }^{(15)}$ and their cancer prevention recommendations $^{(21)}$ suggests consumption of a fibre-rich diet, limiting consumption of foods high in fat, starches or sugars as well as limiting consumption of red and processed meat. Therefore, in this study, the individual food items were collapsed into sixty-four food groups based on their fibre and fat contents, the type of meat or according to their culinary uses. Details on grouping of the foods have been described previously ${ }^{(22)}$. The standard portion sizes were estimated by calculating the average portion size of the individual food items within the food group as per the Food Standards Agency ${ }^{(23)}$.

\section{Case definition}

Incident cases of invasive breast carcinomas, endometrial and ovarian cancers were identified through linkage to the National
Health Service Central Register ${ }^{(24)}$. The International Classification of Diseases 9 and 10 were used to code incident cancer cases. Participants were followed from study entry till diagnosis of the breast cancer (International Classification of Diseases (ICD)-9 code 174 or ICD-10 code C50), endometrial cancer (ICD-9 code 182 or ICD-10 code C54.1 or C54.9), ovarian cancer (ICD-9 code 183 or ICD-10 code C56), date of death or until the censor date (1 April 2016) whichever came first.

\section{Statistical analysis}

Descriptive statistics were used to describe lifestyle characteristics of participants for breast, endometrial and ovarian cancer separately as well as for women without any incident case of a malignant cancer. Cox proportional hazards regression was used to provide hazard ratios (HR) and $99 \%$ CI to account for potential multiple testing of breast, endometrial and ovarian cancers in relation to diet. For ease of interpretation, the HR were presented per standard portion size of the food group per $d$. The proportional hazards assumption was tested graphically as well as using the Cox-Snell residuals for all terms in the model. Time in the study was used as the time variable calculated from the date of questionnaire receipt until either death or censor date.

Risk factors for cancer previously identified in the literature were considered to build a directed acyclic graph. A parsimonious age-adjusted model was firstly used to estimate the association between each individual food groups and risk of the cancers in separate models (model 1). According to the minimal sufficiency set of adjustments, the final models for risk of breast and ovarian cancer were adjusted for age (years), physical activity $(\mathrm{h} / \mathrm{d})^{(25)}$, ethanol intake $(\mathrm{g} / \mathrm{d})^{(26)}$, smoking status (never, current or former smoker) ${ }^{(27)}$, cumulative duration of breastfeeding (weeks) ${ }^{(28-30)}$, menopausal status (pre- or post-menopausal), ${ }^{(2)}$ and socio-economic status (professional/managerial, intermediate or routine and manual) ${ }^{(31)}$ (model 2). For risk of endometrial cancer, history of diabetes ${ }^{(32)}$ and hypertension ${ }^{(33)}$ were also included in model 2. Participants with incomplete data on these variables were excluded.

Subgroup analyses by pre-menopausal cancer and postmenopausal cancer were also performed. A premenopausal cancer was defined as an incident case diagnosed before the last menstrual period, while a postmenopausal cancer case was one diagnosed either at or after the last menstrual period. For premenopausal cancer, cases contributed to person-time from age at baseline until the diagnosis of the event. If the participant did not have a premenopausal cancer, the age until last menstrual period was considered as the time variable instead. Women who were already postmenopausal at study entry were excluded from the model (adjusted for model 2). For postmenopausal cancer, cases contributed to person-time from age at last menstrual period until the diagnosis of the event. Women who were incident cases of premenopausal cancer and those who were still premenopausal at censor date were excluded from the model (adjusted for model 2).

Age at natural menopause was further explored as an effect modifier for the foods that were significantly associated with the risk of the cancers. Previous studies have also demonstrated an 
increased risk of these cancers with a later age at natural menopause due to longer exposure to oestrogen ${ }^{(34)}$. Age at last period was self-reported at both baseline and phase 2. This variable was grouped as having a menopause either between 40 and 49 years $(n 10505)$ or 50 and 65 years ( $n$ 6295). To include only postmenopausal women with a natural menopause, those who had a hysterectomy or bilateral oophorectomy as well as those who reported current or ever use of hormone replacement therapy (HRT) before their last period were excluded from the analyses. In addition, women who had their last period before the age of 40 years were also excluded as this could be due to other treatments or surgical procedures that could not be ascertained in this study. All statistical analyses were conducted using Stata version 15 statistical software.

Sensitivity analysis was also conducted using model 2 , further adjusting for both family history of any cancer and family history of breast cancer in the first-degree relatives to estimate the association between food groups and the risk of breast cancer. To estimate the association of the risk of endometrial cancer, family history of endometrial cancer was included in the model, and for the risk of ovarian cancer, a family history of ovarian cancer and breast cancer was adjusted for in addition to model 2. Sensitivity analyses also involved adjusting for total energy intake $(\mathrm{kJ} / \mathrm{d})$ to account for under- and over-reporters (model 3). Adjustments were also made for current HRT use $^{(35,36)}$, use of oral contraceptive pills and parity ${ }^{(37,38)}$ (model 4) in addition to model 3 as these are known risk factors for breast, endometrial and ovarian cancers.

\section{Results}

\section{Baseline characteristics according to cancer type}

Of the 35372 women at baseline, 695 women who were not flagged on the National Health Services (NHS) digital, 2340 women reporting history of any previous malignant cancer at baseline (except for non-melanoma of the skin) and women who were diagnosed with breast ( $n$ 68), endometrial $(n 7)$ and ovarian ( $n$ 12) cancer within 1 year of baseline were excluded. After the exclusions, 32228 women were eligible for the breast cancer analysis, 32289 for the endometrial cancer analysis and 32284 for the ovarian cancer analysis.

Baseline characteristics of the participants according to cancer type are summarised in Table 1. After approximately 18 years of follow-up, there were 1822 incident cases of breast cancer, 294 and 285 incident cases of endometrial and ovarian cancer, respectively. Women with endometrial and ovarian cancer were on average overweight at baseline with a BMI of 27.3 and $25.1 \mathrm{~kg} / \mathrm{m}^{2}$, respectively, while women with breast cancer were borderline overweight $\left(24.8 \mathrm{~kg} / \mathrm{m}^{2}\right)$ and women without any cancer had a normal weight $\left(24.4 \mathrm{~kg} / \mathrm{m}^{2}\right)$. Women with endometrial cancer were less likely to be current smokers and had lower ethanol intake in comparison to those with breast and ovarian cancer as well as those without any cancer. A majority of women with incident breast cancer were current users of HRT at baseline (58.3\%). Women without any cancer had an earlier natural menopause ( mean $=47.5$ years) as compared with women with breast, endometrial and ovarian cancer. Around $42-46 \%$ of women with breast, endometrial and ovarian cancer had a family history of any cancer at baseline as compared with $38.4 \%$ for the non-cancer cases. Total energy intake and fibre intake was quite similar between the cancer cases and non-cancer cases.

\section{Diet and risk of breast, endometrial and ovarian cancer}

For the association between food intake and risk of breast cancer, in both the age-adjusted model and fully adjusted model, a standard portion of $83 \mathrm{~g}$ of tomato consumption was associated with a significant risk reduction (HR 0.87, 99\% CI $0 \cdot 75,0.999)$. In the fully adjusted model, a standard portion of both processed meat and total meat intake was associated with higher risk of breast cancer, 36 and $17 \%$, respectively (HR 1.36 , $99 \%$ CI 1.02, 1.81; HR 1.17, $99 \%$ CI 1.00, 1.36) (Table 2). According to the subgroup analysis by pre- and postmenopausal breast cancer, consumption of tomatoes reduced the risk of postmenopausal breast cancer but not premenopausal breast cancer. Consumption of processed meat and total meat were both associated with a significant higher risk of postmenopausal breast cancer only. In addition, intake of $15 \mathrm{~g}$ of biscuits per $d$ was associated with a $17 \%$ higher risk of premenopausal breast cancer (Table 3).

Similarly, an increased risk of endometrial cancer was observed in the fully adjusted model with consumption of a standard portion of processed and total meat per d (HR 2.19, $99 \%$ CI 1.34, 3.60; HR 1.53, 99\% CI 1·04, 2.24). Consumptions of $28 \mathrm{~g}$ of dried fruits per $\mathrm{d}$ and $85 \mathrm{~g}$ of high breakfast cereals were associated with a 40 and $26 \%$ reduced risk of endometrial cancer, respectively (HR 0.60, 99\% CI 0.37, 0.97; HR 0.74, 99\% CI $0.55,0.998$ ) (Table 2). In the subgroup analysis, a standard portion of processed meat per $d$ was associated with a higher risk of postmenopausal endometrial cancer. Consumption of dried fruits was associated with a significant reduced risk of only postmenopausal endometrial cancer (HR 0.55, 99\% CI 0.31, 0.98), while a higher intake of low-energy/-diet soft drinks was positively associated with the risk of postmenopausal endometrial cancer (HR 1.27; $99 \%$ CI 1.00, 1.61). For ovarian cancer, $34 \mathrm{~g}$ of mushroom intake per d was associated with a significantly higher risk (HR 1.57, $99 \% 1 \cdot 09,2 \cdot 26$ ). Furthermore, it was found that a higher mushroom intake was associated with an increased risk of postmenopausal ovarian cancer. A higher consumption of citrus fruits and total fruits was associated with an 87 and $37 \%$ reduced risk of premenopausal ovarian cancer, respectively.

After further adjustment for family history of the respective cancers, similar results were obtained to those reported above. In addition, a significantly higher risk of breast and endometrial cancer was observed with frequent consumption of a standard portion of potatoes with added fat (i.e. chips/roast potatoes) (online Supplementary Table S1). The associations between diet and risk of breast, endometrial and ovarian cancer after further adjustments for total energy intake and current HRT use, oral contraceptive use and parity were also in agreement with the study's main associations (online Supplementary Table S2). We also found that the risk of breast, endometrial and ovarian cancer significantly increased with an increase in age at natural 
Table 1. Baseline characteristics from the UK Women's Cohort Study according to cancer type

(Mean values and standard deviations; numbers and percentages)

\begin{tabular}{|c|c|c|c|c|c|c|c|c|}
\hline \multirow[b]{3}{*}{ Characteristics } & \multirow{2}{*}{\multicolumn{2}{|c|}{$\frac{\text { Breast cancer cases }}{n 1822}$}} & \multirow{2}{*}{\multicolumn{2}{|c|}{$\begin{array}{c}\frac{\text { Endometrial cancer cases }}{n 294} \\
\end{array}$}} & \multirow{2}{*}{\multicolumn{2}{|c|}{$\frac{\text { Ovarian cancer cases }}{n 285}$}} & \multirow{2}{*}{\multicolumn{2}{|c|}{$\begin{array}{c}\text { No cancer } \\
n 28929 \\
\end{array}$}} \\
\hline & & & & & & & & \\
\hline & Mean & SD & Mean & SD & Mean & SD & Mean & SD \\
\hline \multicolumn{9}{|l|}{ Demographic characteristics } \\
\hline Age (years) & $53 \cdot 2$ & $9 \cdot 0$ & 54.1 & 8.3 & $55 \cdot 7$ & $9 \cdot 0$ & $51 \cdot 7$ & 9.3 \\
\hline BMI $\left(\mathrm{kg} / \mathrm{m}^{2}\right)$ & $24 \cdot 8$ & 4.3 & $27 \cdot 3$ & $6 \cdot 3$ & $25 \cdot 1$ & 4.5 & $24 \cdot 4$ & $4 \cdot 2$ \\
\hline \multicolumn{9}{|c|}{ Professional/managerial socio-economic status } \\
\hline$n$ & \multicolumn{2}{|c|}{1105} & \multicolumn{2}{|c|}{182} & \multicolumn{2}{|c|}{171} & \multicolumn{2}{|c|}{18262} \\
\hline$\%$ & \multicolumn{2}{|c|}{$62 \cdot 1$} & \multicolumn{2}{|c|}{$63 \cdot 4$} & \multicolumn{2}{|c|}{$61 \cdot 3$} & \multicolumn{2}{|c|}{$63 \cdot 6$} \\
\hline \multirow{2}{*}{\multicolumn{9}{|c|}{$\begin{array}{l}\text { Medical history } \\
\text { Family history of any cancer }\end{array}$}} \\
\hline & & & & & & & & \\
\hline$n$ & \multirow{2}{*}{\multicolumn{2}{|c|}{$\begin{array}{l}755 \\
43.7\end{array}$}} & \multirow{2}{*}{\multicolumn{2}{|c|}{$\begin{array}{l}127 \\
46 \cdot 0\end{array}$}} & \multirow{2}{*}{\multicolumn{2}{|c|}{$\begin{array}{r}112 \\
42.6\end{array}$}} & \multirow{2}{*}{\multicolumn{2}{|c|}{10577}} \\
\hline$\%$ & & & & & & & & \\
\hline \multicolumn{9}{|l|}{ Family history of breast cancer } \\
\hline$n$ & & & & & & & & \\
\hline$\%$ & & & & & & & & \\
\hline Family history of endometrial cancer & & & & & & & & \\
\hline$n$ & & & & & & & & \\
\hline$\%$ & & & & & & & & \\
\hline Family history of ovarian cancer & & & & & & & & \\
\hline$n$ & & & & & & & & \\
\hline$\%$ & & & & & & & & \\
\hline Lifestyle characteristics & & & & & & & & \\
\hline Current smoker & & & & & & & & \\
\hline$n$ & & & & & & & & \\
\hline$\%$ & & & & & & & & \\
\hline Physical activity & 0.25 & 0.55 & 0.24 & 0.44 & 0.19 & 0.34 & 0.26 & 0.49 \\
\hline Reproductive history & & & & & & & & \\
\hline Current hormone replacement therapy & & & & & & & & \\
\hline$n$ & & & & & & & & \\
\hline$\%$ & & & & & & & & \\
\hline Parous & & & & & & & & \\
\hline$n$ & & & & & & & & \\
\hline$\%$ & & & & & & & & \\
\hline Postmenopausal & & & & & & & & \\
\hline$n$ & & & & & & & & \\
\hline$\%$ & & & & & & & & \\
\hline Age at last natural menopause (years) & $48 \cdot 1$ & 4.5 & $50 \cdot 0$ & 4.4 & $49 \cdot 1$ & 3.4 & $47 \cdot 3$ & 4.5 \\
\hline Energy and food intake & & & & & & & & \\
\hline Total energy intake $(\mathrm{kJ} / \mathrm{d})$ & 9586 & 3276 & 9297 & 2992 & 9456 & 2904 & 9586 & 3318 \\
\hline Fibre intake $(g / d)$ & $25 \cdot 5$ & $11 \cdot 2$ & $24 \cdot 2$ & $10 \cdot 3$ & $25 \cdot 4$ & $10 \cdot 1$ & $25 \cdot 6$ & $10 \cdot 9$ \\
\hline Ethanol $(g / d)$ & $9 \cdot 1$ & $10 \cdot 1$ & 7.5 & 8.7 & $9 \cdot 3$ & 11.4 & 8.7 & 10.4 \\
\hline Total vegetable intake $(\mathrm{g} / \mathrm{d})$ & 314.7 & $208 \cdot 7$ & $305 \cdot 0$ & 174.7 & $322 \cdot 8$ & $190 \cdot 6$ & 317.7 & 191.6 \\
\hline Total fruit intake (g/d) & $319 \cdot 1$ & 225.5 & $292 \cdot 4$ & $198 \cdot 3$ & $307 \cdot 2$ & $207 \cdot 7$ & $316 \cdot 1$ & $243 \cdot 3$ \\
\hline Total meat intake $(\mathrm{g} / \mathrm{d})$ & $69 \cdot 1$ & $61 \cdot 2$ & 72.5 & 59.5 & $66 \cdot 3$ & $69 \cdot 3$ & 64.5 & 63.5 \\
\hline
\end{tabular}

menopause (online Supplementary Table S3). Subgroup analysis by age at natural menopause demonstrated that the diet of women with either an earlier or later age at natural menopause did not change the risk of the cancers (online Supplementary Table S4).

\section{Discussion}

In this prospective investigation of the consumption of food groups in relation to the risk of breast, endometrial and ovarian cancers, we consistently found that consumption of processed meat and total meat was associated with a significantly higher risk of breast and endometrial cancer. In addition, frequent consumption of a standard portion of tomatoes and dried fruits were associated with a reduced risk of breast and endometrial cancer, respectively. A higher consumption of mushrooms was found to be weakly associated with a higher risk of ovarian cancer. Subgroup analysis showed similar associations between these food items and cancer risk, when differentiating between a pre- and postmenopausal cancer as well as when further adjustments for family history of cancer, total energy intake, current HRT use, oral contraceptive use and parity were accounted for in the different models.

Previous studies have also reported an increased risk of breast and endometrial cancer with a higher consumption of processed meat and total meat. According to the recent UK Biobank cohort study ${ }^{(39)}$, a $6 \%$ higher risk of breast cancer was reported in relation to processed meat consumption. Similar to our results, they also found only a significant increased risk of postmenopausal breast cancer. The European Prospective Investigation into Cancer and Nutrition (EPIC) $)^{(40)}$ and NutriNet- 
Table 2. Breast, endometrial and ovarian cancer by food groups (Hazard ratios (HR) and $99 \%$ confidence intervals)

\begin{tabular}{|c|c|c|c|c|c|c|c|c|c|c|c|c|}
\hline \multirow[b]{3}{*}{ Daily intake/standard portion size } & \multicolumn{4}{|c|}{ Breast cancer cases } & \multicolumn{4}{|c|}{ Endometrial cancer cases } & \multicolumn{4}{|c|}{ Ovarian cancer cases } \\
\hline & \multicolumn{2}{|c|}{$\begin{array}{c}\text { Model } 1 \\
\left(n 1796 / 32228^{\star}\right)\end{array}$} & \multicolumn{2}{|c|}{$\begin{array}{c}\text { Model } 2 \\
\text { (n 1625/29 183†) }\end{array}$} & \multicolumn{2}{|c|}{$\begin{array}{c}\text { Model } 1 \\
\left(n 285 / 32289^{\star}\right)\end{array}$} & \multicolumn{2}{|c|}{$\begin{array}{c}\text { Model } 2 \\
(n \text { 238/27 338 })\end{array}$} & \multicolumn{2}{|c|}{$\begin{array}{c}\text { Model } 1 \\
\left(n 274 / 32284^{\star}\right)\end{array}$} & \multicolumn{2}{|c|}{$\begin{array}{c}\text { Model } 2 \\
\text { (n 251/29229†) }\end{array}$} \\
\hline & $\mathrm{HR}$ & $99 \% \mathrm{Cl}$ & HR & $99 \% \mathrm{Cl}$ & $\mathrm{HR}$ & $99 \% \mathrm{Cl}$ & $\mathrm{HR}$ & $99 \% \mathrm{Cl}$ & $\mathrm{HR}$ & $99 \% \mathrm{Cl}$ & $\mathrm{HR}$ & $99 \% \mathrm{Cl}$ \\
\hline Starchy food sources & & & & & & & & & & & & \\
\hline Wholegrain products $/ 33 \mathrm{~g}$ & 0.99 & $0.96,1.02$ & 0.99 & $0.96,1.03$ & 0.95 & $0.88,1.03$ & 0.92 & $0.84,1.01$ & 1.02 & $0.94,1.10$ & 1.00 & $0.93,1.09$ \\
\hline Refined grain products $/ 51 \mathrm{~g}$ & 1.03 & $0.96,1.10$ & 1.03 & $0.95,1.11$ & $1 \cdot 11$ & $0.95,1.30$ & $1 \cdot 15$ & $0.98,1.35$ & 1.04 & $0.87,1.24$ & 1.02 & $0.84,1.24$ \\
\hline Low-fibre breakfast cereals/40 g & 1.00 & $0.83,1.20$ & 1.04 & $0.85,1.26$ & 0.83 & $0.50,1.37$ & 0.76 & $0.43,1.37$ & $1 \cdot 16$ & $0.76,1.75$ & 1.08 & $0.67,1.74$ \\
\hline High-fibre breakfast cereals $/ 85 \mathrm{~g}$ & 1.00 & $0.92,1.08$ & 1.01 & $0.92,1.10$ & 0.82 & $0.64,1.06$ & 0.74 & $0.55,0.998$ & 0.89 & $0 \cdot 70,1 \cdot 13$ & 0.89 & $0.69,1.15$ \\
\hline Plain potatoes $/ 210 \mathrm{~g}$ & 0.93 & $0.81,1.06$ & 0.94 & $0.81,1.09$ & 0.92 & $0.66,1.30$ & 0.94 & $0 \cdot 64,1 \cdot 38$ & 0.79 & $0.54,1.15$ & 0.83 & $0.56,1.23$ \\
\hline Potatoes with added fat $/ 127 \mathrm{~g}$ & $1 \cdot 13$ & $0.94,1.37$ & $1 \cdot 28$ & $0.96,1.71$ & $1 \cdot 28$ & $0.97,1.68$ & 1.90 & $1.00,3.60$ & $0 \cdot 78$ & $0.35,1.70$ & 0.80 & $0.35,1.84$ \\
\hline Refined pasta and rice $/ 210 \mathrm{~g}$ & 0.99 & $0.78,1.25$ & 0.94 & $0 \cdot 72,1 \cdot 22$ & 0.99 & $0.55,1.78$ & 1.05 & $0.54,2.05$ & 0.69 & $0.34,1.42$ & 0.73 & $0.34,1.54$ \\
\hline Wholegrain pasta and rice $/ 197 \mathrm{~g}$ & 1.07 & $0.82,1.40$ & $1 \cdot 14$ & $0.84,1.55$ & 0.72 & $0.31,1.67$ & 0.60 & $0.23,1.60$ & 0.58 & $0.23,1.49$ & 0.70 & $0.27,1.83$ \\
\hline rotein and fat food sources & & & & & & & & & & & & \\
\hline Low-fat dairy products $/ 118 \mathrm{~g}$ & 1.01 & $0.98,1.03$ & 1.01 & $0.98,1.03$ & 1.04 & $0.98,1.10$ & 1.03 & $0.97,1.10$ & 0.95 & $0.90,1.02$ & 0.95 & $0.89,1.02$ \\
\hline High-fat dairy products $/ 93 \mathrm{~g}$ & 1.00 & $0.97,1.03$ & 1.00 & $0.97,1.04$ & 0.96 & $0.88,1.04$ & 0.98 & $0.90,1.07$ & 1.05 & $0.98,1.12$ & 1.06 & $0.99,1.13$ \\
\hline Butter and hard margarine $/ 10 \mathrm{~g}$ & 0.99 & $0.93,1.06$ & 0.98 & $0.92,1.05$ & 0.98 & $0.83,1.16$ & 1.00 & $0.83,1.20$ & 0.92 & $0.76,1.10$ & 0.86 & $0.69,1.06$ \\
\hline Margarine $/ 9 \mathrm{~g}$ & 0.97 & $0.91,1.03$ & 0.99 & $0.92,1.05$ & 0.95 & $0.81,1.12$ & 0.93 & $0.77,1.11$ & 1.06 & $0.91,1.22$ & 1.03 & $0.88,1.21$ \\
\hline Low-fat spreads/7g & 1.03 & $0.96,1.09$ & 1.03 & $0.96,1.10$ & 1.02 & $0.87,1.19$ & 0.98 & $0.82,1.17$ & 0.94 & $0.79,1.13$ & 0.95 & $0.78,1 \cdot 15$ \\
\hline High-fat dressing $/ 23 \mathrm{~g}$ & 1.00 & $0.81,1.23$ & 0.98 & $0.78,1 \cdot 22$ & 0.72 & $0.39,1.32$ & 0.77 & $0.40,1.50$ & 0.92 & $0.53,1.61$ & 0.72 & $0.38,1.38$ \\
\hline Low-fat dressing $/ 30 \mathrm{~g}$ & 0.98 & $0.70,1.36$ & 1.02 & $0.72,1.45$ & 0.88 & $0.37,2.08$ & 0.86 & $0.32,2 \cdot 29$ & 1.02 & $0.46,2.30$ & 1.09 & $0.47,2.54$ \\
\hline Soyabean products $/ 62 \mathrm{~g}$ & 0.97 & $0.90,1.04$ & 0.97 & $0.90,1.05$ & 0.98 & $0.82,1.17$ & 0.98 & $0.81,1.19$ & 0.94 & $0.75,1 \cdot 16$ & 0.93 & $0.73,1.19$ \\
\hline Textured vegetable protein $/ 130 \mathrm{~g}$ & 0.44 & $0.03,6.93$ & $0 \cdot 16$ & $0.01,3.50$ & - & - & - & - & - & - & - & - \\
\hline Pulses & 1.00 & $0.87,1.14$ & 1.03 & $0 \cdot 89,1 \cdot 19$ & 0.87 & $0,1 \cdot 28$ & 0.81 & $2,1 \cdot 25$ & 1.08 & 79,1 & $1 \cdot 17$ & $3,1.64$ \\
\hline Eggs/egg dishe & 0.99 & $0.76,1.27$ & 0.98 & $0.73,1.31$ & $1 \cdot 29$ & $0.82,2.02$ & 1.63 & $0.88,2.99$ & $1 \cdot 21$ & $0.74,1.96$ & $1 \cdot 21$ & $0.62,2 \cdot 37$ \\
\hline Fish and fish di & 1.04 & $0.76,1.43$ & 1.01 & $0.68,1.51$ & 0.90 & $0.36,2 \cdot 24$ & 0.96 & $0.34,2 \cdot 71$ & 0.99 & $0.43,2.24$ & 0.86 & $0.30,2.43$ \\
\hline Oily fi & 0.98 & $0.64,1.50$ & 0.98 & $0.62,1.54$ & 0.45 & $0.12,1.68$ & 0.52 & $0.13,2 \cdot 13$ & 1.06 & $0.39,2.89$ & 1.06 & $0.36,3 \cdot 14$ \\
\hline Sh & $1 \cdot 17$ & $0.66,2.07$ & 1.44 & $0.56,3.70$ & 0.52 & $0.04,6.83$ & 0.72 & $0.04,11.69$ & 0.85 & $0.11,6.65$ & 0.65 & $0.04,10.06$ \\
\hline Red $\mathrm{m}$ & $1 \cdot 20$ & $0.97,1.49$ & $1 \cdot 28$ & $0.95,1.72$ & 1.33 & $0.87,2.02$ & 1.90 & $0.92,3.94$ & 0.91 & $0.45,1.88$ & 0.85 & $0.38,1.92$ \\
\hline meat $/ 74 \mathrm{~g}$ & $1 \cdot 34$ & $1.03,1.73$ & 1.36 & $1.02,1.81$ & 1.81 & $1 \cdot 16,2 \cdot 83$ & $2 \cdot 19$ & $1 \cdot 34,3 \cdot 60$ & $1 \cdot 22$ & $0.62,2.42$ & 1.27 & $0.60,2.69$ \\
\hline Poultry & $1 \cdot 30$ & $0.90,1.87$ & $1 \cdot 32$ & $0.86,2.03$ & 1.35 & $0.55,3.32$ & 1.76 & $0.60,5 \cdot 18$ & 0.63 & $0.19,2.07$ & 0.62 & $0 \cdot 17,2 \cdot 21$ \\
\hline Offal/1 & $2 \cdot 19$ & $0.44,10.89$ & $2 \cdot 27$ & $0.41,12.55$ & $2 \cdot 70$ & $0.05,138.5$ & - & - & $0 \cdot 10$ & $0.00,12.00$ & 0.07 & $0.00,12.00$ \\
\hline Total meat/1 & $1 \cdot 12$ & $1.01,1 \cdot 24$ & $1 \cdot 17$ & $1 \cdot 00,1.36$ & $1 \cdot 19$ & $0.98,1.45$ & 1.53 & $1 \cdot 04,2 \cdot 24$ & 0.94 & $0.65,1.37$ & 0.92 & $0.61,1.39$ \\
\hline & & & & & & & & & & & & \\
\hline Vegetable dis & 0.97 & $1 \cdot 14$ & 0.91 & 0.75 & 0.74 & 22 & 0.67 & 19 & 1.02 & 0.70 & 1.03 & $4,1.67$ \\
\hline Alliums $/ 39 \mathrm{~g}$ & 0.98 & $0.82,1.17$ & 0.99 & $0.82,1 \cdot 20$ & 1.02 & $0.67,1.57$ & 0.97 & $0.58,1.61$ & $0 \cdot 81$ & $0.49,1.33$ & 0.77 & $0.44,1.33$ \\
\hline Fresh legumes & 1.01 & $0.86,1.18$ & 0.96 & $0.80,1 \cdot 15$ & $1 \cdot 12$ & $0.80,1.56$ & $1 \cdot 14$ & $0.75,1.72$ & 1.03 & $0.71,1.51$ & 1.08 & $0.73,1.60$ \\
\hline vegetables $/ 60 \mathrm{~g}$ & 0.98 & $0 \cdot 87,1 \cdot 10$ & 0.96 & $0.84,1.09$ & 0.98 & $0.73,1.32$ & 0.85 & $0.58,1.23$ & $1 \cdot 17$ & $0.93,1.47$ & $1 \cdot 18$ & $0.90,1.56$ \\
\hline $143 \mathrm{~g}$ & 0.97 & $0.87,1.08$ & 0.97 & $0.87,1.09$ & 0.84 & $0.62,1.12$ & 0.84 & $0.61,1.17$ & 0.98 & $0.76,1.28$ & 0.99 & $0.74,1.32$ \\
\hline getables $/ 75 \mathrm{~g}$ & 1.01 & $0.95,1.07$ & 0.99 & $0.91,1.06$ & 0.94 & $0.78,1.14$ & 0.94 & $0.76,1.16$ & 1.01 & $0.87,1.18$ & 1.04 & $0.88,1.24$ \\
\hline & 0.88 & $0.77,1.00$ & 0.87 & $0.75,0.999$ & 0.81 & $0.57,1.15$ & 0.77 & $0.52,1.16$ & 0.94 & $0.69,1.29$ & 0.97 & $0.70,1.35$ \\
\hline Mushroon & 0.98 & $0.79,1 \cdot 22$ & 0.96 & $0.76,1 \cdot 22$ & $1 \cdot 19$ & $0.77,1.85$ & $1 \cdot 29$ & $0.78,2 \cdot 12$ & 1.40 & $0.98,1.99$ & 1.57 & $1 \cdot 09,2 \cdot 26$ \\
\hline Root & 0.94 & $0.83,1.05$ & 0.94 & $0.83,1.06$ & 0.96 & $0.74,1.25$ & 0.90 & $0.66,1.25$ & 1.06 & $0.83,1.34$ & $1 \cdot 12$ & $0.88,1.43$ \\
\hline Total vegetables $/ 150 \mathrm{~g}$ & 0.98 & $0.94,1.03$ & 0.97 & $0.91,1.02$ & 0.95 & $0.84,1.09$ & 0.93 & $0.80,1.08$ & 1.02 & $0.91,1.14$ & 1.04 & $0.92,1.18$ \\
\hline & & & & & & & & & & & & \\
\hline Ston & 1.00 & 1.04 & 1.03 & $0.86,1 \cdot 23$ & 0.84 & & 0.94 & 1.62 & 0.63 & $0.32,1.22$ & 0.66 & $2,1 \cdot 33$ \\
\hline and yellow fruits $/ 118 \mathrm{~g}$ & 1.03 & $0.90,1.18$ & 1.08 & $0.92,1.26$ & 0.67 & $0.39,1 \cdot 15$ & 0.75 & $1 \cdot 32$ & 0.97 & $0.65,1.44$ & 0.98 & $0.62,1.54$ \\
\hline $100 \mathrm{~g}$ & 0.98 & $0.86,1 \cdot 11$ & 0.96 & $0.84,1 \cdot 10$ & 0.92 & $0.66,1.29$ & 0.91 & $0.61,1.34$ & 0.84 & $0.57,1.23$ & 0.91 & $0.62,1.32$ \\
\hline Citrus family fruits $/ 92 \mathrm{~g}$ & 1.03 & $0.93,1.14$ & 1.02 & $0.92,1.14$ & 0.81 & $0.60,1.11$ & 0.77 & $0.54,1.10$ & 0.85 & $0.63,1.15$ & 0.88 & $0.64,1.21$ \\
\hline Rhubarb/130 g & 0.96 & $0.76,1.22$ & 0.93 & $0.71,1.24$ & 0.59 & $0.24,1.45$ & 0.74 & $0.30,1.82$ & 1.04 & $0.61,1.77$ & 1.07 & $0.57,2.00$ \\
\hline Berries/48 g & 1.02 & $0.93,1.11$ & 1.03 & $0.94,1.14$ & 0.85 & $0.62,1.15$ & 0.85 & $0.60,1 \cdot 21$ & 0.84 & $0.61,1.15$ & 0.82 & $0.57,1.17$ \\
\hline $\mathrm{s} / 100 \mathrm{~g}$ & 1.04 & $0.94,1.158$ & 1.07 & $0.95,1.19$ & 0.87 & $0.65,1.18$ & 0.88 & $0.63,1.22$ & $1 \cdot 10$ & $0.85,1.42$ & $1 \cdot 21$ & $0.92,1.59$ \\
\hline Pomes/116g & 0.97 & $0.90,1.04$ & 0.98 & $0.91,1.06$ & 0.97 & $0.80,1 \cdot 16$ & 0.92 & $0.75,1.15$ & 0.91 & $0.74,1.11$ & 0.97 & $0.79,1.19$ \\
\hline Total fruits $/ 150 \mathrm{~g}$ & 1.00 & $0.96,1.04$ & 1.01 & $0.97,1.05$ & 0.91 & $0.81,1.02$ & 0.90 & $0.79,1.03$ & 0.95 & $0.85,1.06$ & 0.98 & $0.88,1.10$ \\
\hline Dried fruits $/ 28 \mathrm{~g}$ & 1.03 & $0.96,1.11$ & 1.04 & $0.98,1.13$ & 0.67 & $0.46,0.99$ & 0.60 & $0.37,0.97$ & 1.02 & $0.86,1.22$ & 1.06 & $0.89,1.26$ \\
\hline Other food groups & & & & & & & & & & & & \\
\hline Sauces/83g & 1.05 & & 1.07 & $0.62,1.87$ & 1.46 & $3 \cdot 40$ & $1 \cdot 29$ &, $5 \cdot 37$ & 1.48 & $0.49,4.49$ & 1.78 & 6.65 \\
\hline Pickles/chutneys/35 g & 0.90 & $0.70,1 \cdot 17$ & 0.89 & $0.68,1.18$ & $1 \cdot 16$ & $0.68,1.97$ & 0.96 & $0.49,1.91$ & 0.72 & $0.35,1.48$ & 0.65 & $0.29,1.44$ \\
\hline Soups/163g & 0.98 & $0.82,1 \cdot 18$ & 0.98 & $0.79,1 \cdot 22$ & 0.93 & $0.57,1.51$ & 0.90 & $0.50,1.61$ & 0.95 & $0.60,1.50$ & 1.03 & $0.62,1.70$ \\
\hline Confectionery and spreads/44 g & 0.98 & $0.92,1.04$ & 0.99 & $0.92,1.05$ & 0.94 & $0.79,1.12$ & 0.88 & $0.71,1.09$ & 0.98 & $0.83,1.15$ & 0.96 & $0.81,1 \cdot 15$ \\
\hline Nuts and seeds $/ 24 \mathrm{~g}$ & 1.01 & $0.93,1.10$ & 1.03 & $0.94,1 \cdot 13$ & 1.03 & $0.85,1.25$ & 0.77 & $0.53,1.13$ & 1.02 & $0.83,1.25$ & 1.02 & $0.80,1.30$ \\
\hline Savoury snacks/26g & 1.05 & $0.87,1.26$ & 1.06 & $0.87,1.29$ & $1 \cdot 21$ & $0.79,1.85$ & $1 \cdot 12$ & $0.68,1.86$ & 1.05 & $0.63,1.73$ & 1.06 & $0.63,1.81$ \\
\hline Biscuits/15 g & 1.00 & $0.94,1.06$ & 1.01 & $0.94,1.08$ & 0.97 & $0.83,1.14$ & 0.97 & $0.81,1.17$ & 0.95 & $0.80,1.13$ & 0.95 & $0.80,1 \cdot 15$ \\
\hline Cakes $/ 66 \mathrm{~g}$ & 0.89 & $0.68,1.16$ & $0 \cdot 88$ & $0.65,1.19$ & 0.85 & $0.43,1.68$ & 0.84 & $0.38,1.87$ & 1.01 & $0.55,1.83$ & 0.95 & $0.47,1.92$ \\
\hline Pastries and puddings $/ 84 \mathrm{~g}$ & 1.05 & $0.89,1.24$ & $1 \cdot 12$ & $0.92,1.36$ & 0.85 & $0.51,1.43$ & 1.00 & $0.58,1.73$ & 0.78 & $0.45,1.35$ & 0.71 & $0.37,1.34$ \\
\hline . & & & & & & & & & & & & \\
\hline $\mathrm{Tea} / 260 \mathrm{~g}$ & 0.98 & $0.95,1.02$ & 0.98 & $0.95,1.02$ & 1.04 & $0.96,1.12$ & 1.02 & $0.93,1.11$ & 0.98 & $0.91,1.07$ & 0.98 & $0.90,1.07$ \\
\hline
\end{tabular}


Table 2. Continued

\begin{tabular}{|c|c|c|c|c|c|c|c|c|c|c|c|c|}
\hline \multirow[b]{3}{*}{ Daily intake/standard portion size } & \multicolumn{4}{|c|}{ Breast cancer cases } & \multicolumn{4}{|c|}{ Endometrial cancer cases } & \multicolumn{4}{|c|}{ Ovarian cancer cases } \\
\hline & \multicolumn{2}{|c|}{$\begin{array}{c}\text { Model } 1 \\
\left(n 1796 / 32228^{\star}\right)\end{array}$} & \multicolumn{2}{|c|}{$\begin{array}{c}\text { Model } 2 \\
(n 1625 / 29183 \dagger) \\
\end{array}$} & \multicolumn{2}{|c|}{$\begin{array}{c}\text { Model 1 } \\
(n \text { 285/32289*) }\end{array}$} & \multicolumn{2}{|c|}{$\begin{array}{c}\text { Model } 2 \\
\text { (n 238/27338 } \ddagger) \\
\end{array}$} & \multicolumn{2}{|c|}{$\begin{array}{c}\text { Model } 1 \\
\left(n 274 / 32284^{*}\right) \\
\end{array}$} & \multicolumn{2}{|c|}{$\begin{array}{c}\text { Model } 2 \\
\text { (n 251/29229†) } \\
\end{array}$} \\
\hline & $\mathrm{HR}$ & $99 \% \mathrm{Cl}$ & $\mathrm{HR}$ & $99 \% \mathrm{Cl}$ & HR & $99 \% \mathrm{Cl}$ & HR & $99 \% \mathrm{Cl}$ & $\mathrm{HR}$ & $99 \% \mathrm{Cl}$ & $\mathrm{HR}$ & $99 \% \mathrm{Cl}$ \\
\hline Herbal tea/260 g & 0.97 & $0.90,1.04$ & 0.99 & $0.91,1.06$ & 0.96 & $0.80,1 \cdot 16$ & 0.89 & $0.71,1 \cdot 12$ & 0.94 & $0.77,1.15$ & 0.93 & $0.75,1 \cdot 16$ \\
\hline Coffee/190 g & 1.01 & $0.98,1.04$ & 1.01 & $0.97,1.04$ & 1.03 & $0.95,1.12$ & 1.03 & $0.94,1.13$ & 1.04 & $0.96,1.13$ & 1.04 & $0.95,1.13$ \\
\hline Other hot beverages $/ 23 \mathrm{~g}$ & 1.02 & $0.92,1.12$ & 1.03 & $0.93,1.14$ & 1.03 & $0.81,1.31$ & 1.01 & $0.77,1.33$ & 0.99 & $0.77,1.28$ & 1.04 & $0.80,1.35$ \\
\hline Juices $/ 145 \mathrm{~g}$ & 1.00 & $0.93,1.07$ & 1.01 & $0.93,1.08$ & 0.97 & $0.80,1.16$ & 0.95 & $0 \cdot 76,1 \cdot 17$ & 0.95 & $0.78,1 \cdot 15$ & 0.97 & $0.79,1.18$ \\
\hline Soft drinks $/ 111 \mathrm{~g}$ & 1.00 & $0.89,1.10$ & 1.00 & $0.90,1.12$ & 1.05 & $0.83,1.33$ & 1.00 & $0.74,1.34$ & 1.03 & $0.80,1.33$ & 1.02 & $0.78,1.33$ \\
\hline Low energy/diet soft drinks/161 g & 1.01 & $0.91,1 \cdot 12$ & 1.03 & $0.93,1.14$ & $1 \cdot 10$ & $0.87,1.38$ & 1.03 & $0.79,1.35$ & 0.96 & $0.72,1.28$ & 0.98 & $0.73,1.31$ \\
\hline Wines/glass§ & 1.03 & $0.94,1.12$ & 1.03 & $0.94,1.13$ & 0.90 & $0.70,1.14$ & 0.90 & $0.69,1.17$ & 1.06 & $0.86,1.32$ & 1.06 & $0.85,1.32$ \\
\hline Beer and cider/half pint§ & 1.09 & $0.93,1.28$ & $1 \cdot 10$ & $0.93,1.29$ & $1 \cdot 13$ & $0.77,1.68$ & 0.81 & $0.42,1.56$ & $1 \cdot 11$ & $0.71,1.72$ & $1 \cdot 10$ & $0.72,1.69$ \\
\hline Port, sherry, liqueurs/glass§ & 0.97 & $0.75,1.26$ & 0.98 & $0.74,1.29$ & 0.93 & $0.47,1.82$ & $1 \cdot 11$ & $0.57,2.17$ & $1 \cdot 17$ & $0.72,1.92$ & 1.20 & $0.74,1.95$ \\
\hline Spirits/measure§ & $1 \cdot 11$ & $0.97,1.27$ & $1 \cdot 10$ & $0.95,1.27$ & 0.51 & $0.25,1.02$ & 0.54 & $0.26,1 \cdot 12$ & $1 \cdot 27$ & $0.97,1.67$ & 1.26 & $0.96,1.66$ \\
\hline
\end{tabular}

* Model 1: adjusted for age.

† Model 2: adjusted for age, ethanol intake, duration of breast-feeding, physical activity, smoking, social class and menopausal status.

$\ddagger$ Model 2 (endometrial cancer): adjusted for age, ethanol intake, duration of breast-feeding, physical activity, smoking, social class, menopausal status, history of diabetes and history of hypertension.

$\S$ Not adjusted for ethanol intake.

Santé ${ }^{(41)}$ prospective cohort studies have also reported an increased risk of breast cancer associated with the consumption of processed meat. Our findings are further supported by a prospective randomised control trial conducted over a period of 8 years $^{(42)}$. Studies investigating the association between processed meat and the risk of endometrial cancer are limited and conflicting. While a case-control study ${ }^{(43)}$ including 274 participants with endometrial cancer found that intake of processed meats such as boiled ham, salami and sausages and canned meat was associated with an increased risk of endometrial cancer, findings from a cohort study, the National Institutes of Health - American Association of Retired Persons (NIH-AARP) Diet and Health Study ${ }^{(44)}$ including 1486 incident cases reported no evidence of an association. Another cancer multi-site study from the NIH-AARP Diet and Health Study also reported no association between processed meat consumption and risk of both breast and endometrial cancer ${ }^{(45)}$.

The underlying mechanisms for the pathogenesis of breast cancer are heterogeneous. High levels of nitrates, nitrites and amines, which are precursors of $\mathrm{N}$-nitroso compounds, added in processed meat to enhance its colour and flavour have been consistently reported to be one of the causes of carcinogenicity ${ }^{(46)}$. In addition, cooking especially at high temperatures (e.g. frying, grilling or barbecuing) can lead to the formation of heterocyclic aromatic amines, which are also potent mutagens and carcinogens ${ }^{(47)}$. The $N$-nitro compounds, heterocyclic amines along with other compounds (haem Fe, saturated fat and oestradiol), present in meats can directly cause DNA damage and have been associated with mammary tumour development as demonstrated in both animal and human studies $^{(46,48)}$. We also found that processed meat consumption was positively associated with postmenopausal breast cancer though not for premenopausal breast cancer. Disparities could be due to differing oestrogen metabolism pathways between the two groups. These results could suggest that processed meat influences breast cancer risk by interacting with oestrogen metabolism in scenarios where the levels of circulating oestrogens are lower ${ }^{(20)}$.
Endometrial cancer is a hormone-driven cancer, with approximately $80 \%$ potentially arising due to either an excess of oestrogen or a lack of progesterone. In the normal endometrium, the proliferative effects of oestrogen are normally countered by progesterone but in the absence of progesterone, oestrogen can induce oncogenesis, an effect that is amplified in situations of excess oestrogen ${ }^{(49)}$. In addition to being a source of $N$-nitroso compounds, processed meat is also rich in cholesterol, which can be converted into androgens and oestrogens through varying metabolic pathways ${ }^{(50)}$.

Our study further demonstrated that consumption of a standard portion of tomatoes per $\mathrm{d}$ was associated with a reduced risk of breast cancer. The protective association was mainly observed among women with postmenopausal breast cancer. Lycopene, a carotenoid widely available in tomatoes, has a very high antioxidant potential and can thus protect the DNA from damage. In a large pooled analysis which included more than 3000 breast cancer cases, Eliassen et al. ${ }^{(51)}$ also found an inverse association between lycopene and risk of breast cancer. The anti-proliferative effect of lycopene has also been demonstrated in mammary cancer cell lines by its inhibitory effect on insulin-like growth factor-I-stimulated cell multiplying ${ }^{(52,53)}$. The observed inverse association could also be due to the high flavonol content of tomatoes which also confers enhanced antioxidant capacity.

Consumption of dried fruits and high-fibre breakfast cereals such as porridge, muesli and bran flakes were inversely associated with risk of endometrial cancer, in particularly among women who were incident cases of postmenopausal endometrial cancer. Dried fruits reportedly have a higher total phenolic content, flavonoids and total antioxidant capacity compared with fresh fruits, making dried fruits a potential candidate of a chemopreventive food ${ }^{(54,55)}$. Previous studies have similarly reported an inverse association between wholegrain cereal consumption and endometrial cancer ${ }^{(56,57)}$. Dietary fibre has been found to interact with the metabolism of oestrogen, causing a reduced bioavailability of the hormone ${ }^{(58)}$. High-fibre cereals and dried fruits are also good sources of 
Table 3. Associations between various food groups and risk of breast, endometrial and ovarian cancer by incidence of premenopausal and postmenopausal cancer cases (Hazard ratios (HR) and $99 \%$ confidence intervals)

\begin{tabular}{|c|c|c|c|c|c|c|c|c|c|c|c|c|}
\hline \multirow{3}{*}{$\begin{array}{l}\text { Daily intake/standard portion } \\
\text { size }\end{array}$} & \multicolumn{4}{|c|}{ Breast cancer cases* } & \multicolumn{4}{|c|}{ Endometrial cancer cases $†$} & \multicolumn{4}{|c|}{ Ovarian cancer cases ${ }^{*}$} \\
\hline & \multicolumn{2}{|c|}{$\begin{array}{l}\text { Premenopausal } \\
\text { (n 291/3178) }\end{array}$} & \multicolumn{2}{|c|}{$\begin{array}{l}\text { Postmenopausal } \\
\text { ( } n \text { 1030/23 806) }\end{array}$} & \multicolumn{2}{|c|}{$\begin{array}{l}\text { Premenopausal } \\
\quad(n 35 / 3024)\end{array}$} & \multicolumn{2}{|c|}{$\begin{array}{l}\text { Postmenopausal } \\
(n 175 / 24118)\end{array}$} & \multicolumn{2}{|c|}{$\begin{array}{l}\text { Premenopausal } \\
\quad(n 44 / 3030)\end{array}$} & \multicolumn{2}{|c|}{$\begin{array}{l}\text { Postmenopausal } \\
\text { ( } n \text { 163/24 115) }\end{array}$} \\
\hline & $\mathrm{HR}$ & $99 \% \mathrm{Cl}$ & $\mathrm{HR}$ & $99 \% \mathrm{Cl}$ & $\mathrm{HR}$ & $99 \% \mathrm{Cl}$ & $\mathrm{HR}$ & $99 \% \mathrm{Cl}$ & $\mathrm{HR}$ & $99 \% \mathrm{Cl}$ & $\mathrm{HR}$ & $99 \% \mathrm{Cl}$ \\
\hline \multicolumn{13}{|l|}{ Starchy food sources } \\
\hline Wholegrain products $/ 33 \mathrm{~g}$ & 1.01 & $0.93,1.10$ & 0.98 & $0.94,1.02$ & $1 \cdot 21$ & $0.84,1.76$ & 0.91 & $0.81,1.01$ & $1 \cdot 21$ & $0.94,1.56$ & 1.01 & $0.91,1.12$ \\
\hline Refined grain products $/ 51 \mathrm{~g}$ & 0.99 & $0.83,1.18$ & 1.06 & $0.97,1.16$ & 1.06 & $0.55,2.03$ & $1 \cdot 16$ & $0.95,1.42$ & 1.31 & $0.90,1.91$ & 0.84 & $0.63,1.13$ \\
\hline $\begin{array}{l}\text { Low-fibre breakfast cereals/ } \\
40 \mathrm{~g}\end{array}$ & 0.90 & $0.60,1.34$ & 1.02 & $0.80,1.30$ & 0.29 & $0.06,1.50$ & 0.75 & $0.38,1.50$ & 0.68 & $0.17,2 \cdot 80$ & 1.02 & $0.55,1.86$ \\
\hline $\begin{array}{l}\text { High-fibre breakfast cereals/ } \\
85 \mathrm{~g}\end{array}$ & 1.06 & $0.87,1.29$ & 1.00 & $0.90,1.12$ & 1.34 & $0.41,4.42$ & 0.86 & $0 \cdot 62,1 \cdot 17$ & 1.28 & $0.63,2.58$ & 0.86 & $0.62,1.19$ \\
\hline Plain potatoes $/ 210 \mathrm{~g}$ & 0.98 & $0.61,1.56$ & 0.95 & $0.79,1.14$ & 0.58 & $0.09,3.69$ & 0.98 & $0.63,1.51$ & 0.97 & $0.27,3.47$ & 0.86 & $0.53,1.40$ \\
\hline Potatoes with added fat $/ 127 \mathrm{~g}$ & 1.05 & $0.49,2.27$ & 1.31 & $0.90,1.91$ & 0.33 & $0.03,3.29$ & 1.96 & $0.89,4.31$ & $9 \cdot 87$ & $0.87,111.5$ & 0.54 & $0.17,1.70$ \\
\hline Refined pasta and rice $/ 210 \mathrm{~g}$ & $1 \cdot 10$ & $0.60,2.01$ & 1.00 & $0.71,1.40$ & 1.04 & $0.13,8.14$ & 1.28 & $0.62,2.63$ & 2.91 & $0.37,22.9$ & 0.72 & $0.28,1.88$ \\
\hline $\begin{array}{l}\text { Wholegrain pasta and rice/ } \\
197 \mathrm{~g}\end{array}$ & $1 \cdot 15$ & $0.49,2 \cdot 70$ & 1.29 & $0.88,1.88$ & 4.90 & $0.51,47 \cdot 3$ & 0.44 & $0.12,1.56$ & 0.06 & $0.00,3.08$ & 1.32 & $0.51,3.42$ \\
\hline \multicolumn{13}{|l|}{ Protein and fat food sources } \\
\hline Low-fat dairy products $/ 118 \mathrm{~g}$ & 1.03 & $0.97,1 \cdot 10$ & 1.02 & $0.99,1.05$ & 0.96 & $0.81,1.14$ & 1.04 & $0.97,1.12$ & 1.02 & $0.85,1.22$ & 0.96 & $0.89,1.04$ \\
\hline High-fat dairy products $/ 93 \mathrm{~g}$ & 1.00 & $0.93,1.08$ & 1.00 & $0.96,1.04$ & 0.94 & $0.73,1.20$ & 0.93 & $0.82,1.05$ & 1.05 & $0.89,1.23$ & 1.04 & $0.95,1.13$ \\
\hline $\begin{array}{l}\text { Butter and hard margarine/ } \\
10 \mathrm{~g}\end{array}$ & 1.00 & $0.82,1.21$ & 0.99 & $0.91,1.09$ & 1.04 & $0.50,2.17$ & 1.02 & $0.83,1.26$ & 0.90 & $0.57,1.43$ & 0.76 & $0.57,1.03$ \\
\hline Margarine $/ 9 \mathrm{~g}$ & 1.08 & $0.91,1.28$ & 0.98 & $0.90,1.06$ & 0.75 & $0.42,1.33$ & 0.93 & $0.75,1.15$ & 1.06 & $0.67,1.68$ & 1.08 & $0.90,1.31$ \\
\hline Low-fat spreads $/ 7 \mathrm{~g}$ & 1.03 & $0.90,1.18$ & 0.98 & $0.90,1.07$ & $1 \cdot 15$ & $0.64,2.06$ & 0.98 & $0.80,1.21$ & 1.46 & $0.84,2.55$ & 0.94 & $0.74,1.19$ \\
\hline High-fat dressing $/ 23 \mathrm{~g}$ & 1.39 & $0.69,2.82$ & 1.00 & $0.76,1.33$ & 0.25 & $0.01,4.55$ & 0.84 & $0.40,1.78$ & 0.34 & $0.05,2.49$ & 0.99 & $0.48,2.02$ \\
\hline Low-fat dressing $/ 30 \mathrm{~g}$ & 1.06 & $0.41,2.71$ & 0.99 & $0.64,1.53$ & 0.64 & $0.02,24.3$ & 0.87 & $0.29,2.62$ & 3.31 & $0.06,175 \cdot 2$ & 1.26 & $0.49,3.23$ \\
\hline Soyabean products $/ 62 \mathrm{~g}$ & 0.90 & $0.69,1 \cdot 17$ & 0.99 & $0.90,1.08$ & 0.84 & $0.42,1.65$ & 1.02 & $0.84,1.25$ & - & - & 0.91 & $0.66,1.25$ \\
\hline $\begin{array}{l}\text { Textured vegetable protein/ } \\
130 \mathrm{~g}\end{array}$ & - & - & 0.04 & $0.00,2.55$ & - & - & - & - & - & - & - & - \\
\hline Pulses $/ 91 \mathrm{~g}$ & 1.04 & $0.71,1.53$ & 1.06 & $0.88,1.27$ & 0.82 & $0.23,2.90$ & 0.90 & $0.55,1.48$ & 1.31 & $0.44,3.89$ & $1 \cdot 28$ & $0.84,1.94$ \\
\hline Eggs/egg dishes $/ 88 \mathrm{~g}$ & 0.92 & $0.44,1.95$ & 0.94 & $0.65,1.37$ & $1 \cdot 18$ & $0.07,18.4$ & 1.64 & $0.84,3.21$ & 0.70 & $0.14,3 \cdot 64$ & 0.86 & $0.33,2.22$ \\
\hline Fish and fish dishes $/ 140 \mathrm{~g}$ & 0.84 & $0.29,2.38$ & 1.01 & $0.61,1.67$ & 1.88 & $0.07,51 \cdot 3$ & 0.81 & $0.23,2.91$ & 0.56 & $0.01,31.7$ & 1.04 & $0.30,3.58$ \\
\hline Oily fish/90 g & 0.46 & $0.11,1.81$ & 0.93 & $0.52,1.63$ & 0.46 & $0.00,104.8$ & 0.27 & $0.04,1.64$ & 0.21 & $0.01,6.52$ & 0.95 & $0.24,3.82$ \\
\hline Shell fish $/ 60 \mathrm{~g}$ & 0.83 & $0.04,17.7$ & 2.06 & $0.64,6 \cdot 61$ & - & - & 0.25 & $0.01,11.5$ & - & - & 1.39 & $0.06,33.7$ \\
\hline Red meat $/ 189 \mathrm{~g}$ & 0.91 & $0.40,2.05$ & 1.37 & $0.94,1.98$ & 0.44 & $0.04,5.37$ & 1.86 & $0.80,4.30$ & $2 \cdot 55$ & $0.66,9.77$ & 0.62 & $0.21,1.80$ \\
\hline Processed meat $/ 74 \mathrm{~g}$ & 1.36 & $0.66,2.80$ & 1.50 & $1.01,2.22$ & 0.65 & $0.03,12 \cdot 1$ & 3.05 & $1.34,6.91$ & $2 \cdot 13$ & $0.84,5.40$ & 0.71 & $0.23,2 \cdot 18$ \\
\hline Poultry $/ 143 \mathrm{~g}$ & 1.08 & $0.33,3.55$ & 1.33 & $0.78,2.28$ & - & - & 1.29 & $0.35,4.81$ & - & - & 0.54 & $0.11,2.66$ \\
\hline $\mathrm{Offal} / 100 \mathrm{~g}$ & - & - & 3.67 & $0.49,27 \cdot 2$ & - & - & - & - & _- & _- & 0.05 & $0.00,30.6$ \\
\hline Total meat $/ 150 \mathrm{~g}$ & 1.03 & $0.69,1.56$ & 1.22 & $1.00,1.47$ & 0.94 & $0.27,3.26$ & 1.50 & $0.95,2.35$ & 1.67 & $0.89,3 \cdot 13$ & 0.75 & $0.44,1.29$ \\
\hline \multicolumn{13}{|l|}{ Vegetables } \\
\hline Vegetable dishes $/ 214 \mathrm{~g}$ & 1.00 & $0.60,1.67$ & 1.00 & $0.79,1.27$ & 1.73 & $0.39,7.72$ & 0.77 & $0.40,1.48$ & 0.36 & $0.08,1.70$ & 1.23 & $0.72,2 \cdot 10$ \\
\hline Alliums $/ 39 \mathrm{~g}$ & 0.81 & $0.46,1.42$ & $1 \cdot 12$ & $0.91,1.37$ & 1.28 & $0.33,5.03$ & 0.95 & $0.53,1.72$ & 0.32 & $0.08,1.33$ & 0.96 & $0.53,1.74$ \\
\hline Fresh legumes $/ 75 \mathrm{~g}$ & 0.87 & $0.49,1.56$ & 1.09 & $0.89,1.33$ & 1.91 & $0.39,9.24$ & 1.23 & $0.79,1.90$ & 0.54 & $0.12,2.40$ & 1.21 & $0.78,1.87$ \\
\hline $\begin{array}{l}\text { Mediterranean vegetables/ } \\
60 \mathrm{~g}\end{array}$ & 0.98 & $0.65,1.50$ & 1.04 & $0.89,1.22$ & 1.24 & $0.51,3.00$ & 0.93 & $0.61,1.42$ & 0.54 & $0.21,1.35$ & 1.23 & $0.88,1.72$ \\
\hline Salad vegetables $/ 43 \mathrm{~g}$ & 0.99 & $0.68,1.44$ & 1.04 & $0.91,1.20$ & 1.34 & $0.37,4.87$ & 0.88 & $0.61,1.28$ & 0.61 & $0.28,1.31$ & 1.00 & $0.70,1.43$ \\
\hline Cruciferous vegetables $/ 75 \mathrm{~g}$ & 0.94 & $0.74,1.20$ & 1.03 & $0.94,1.12$ & 1.09 & $0.46,2.60$ & 0.97 & $0.77,1.23$ & 1.06 & $0.58,1.93$ & $1 \cdot 10$ & $0.93,1.30$ \\
\hline Tomatoes $/ 83 \mathrm{~g}$ & 0.96 & $0.62,1.48$ & 0.88 & $0.74,1.04$ & 1.85 & $0.61,5.62$ & 0.76 & $0.48,1.22$ & $1 \cdot 16$ & $0.54,2.49$ & 0.91 & $0.59,1.39$ \\
\hline Mushrooms $/ 34 \mathrm{~g}$ & 0.94 & $0.51,1.75$ & 1.03 & $0.77,1.38$ & $2 \cdot 13$ & $0.26,14 \cdot 7$ & 1.24 & $0.66,2.31$ & 0.29 & $0.06,1.43$ & 1.84 & $1 \cdot 21,2 \cdot 79$ \\
\hline
\end{tabular}




\begin{tabular}{|c|c|c|c|c|c|c|c|c|c|c|c|c|}
\hline \multirow{3}{*}{$\begin{array}{l}\text { Daily intake/standard portion } \\
\text { size }\end{array}$} & \multicolumn{4}{|c|}{ Breast cancer cases ${ }^{*}$} & \multicolumn{4}{|c|}{ Endometrial cancer cases $\dagger$} & \multicolumn{4}{|c|}{ Ovarian cancer cases* } \\
\hline & \multicolumn{2}{|c|}{$\begin{array}{l}\text { Premenopausal } \\
(n \text { 291/3178) }\end{array}$} & \multicolumn{2}{|c|}{$\begin{array}{l}\text { Postmenopausal } \\
\text { (n 1030/23 806) }\end{array}$} & \multicolumn{2}{|c|}{$\begin{array}{l}\text { Premenopausal } \\
\quad(n 35 / 3024)\end{array}$} & \multicolumn{2}{|c|}{$\begin{array}{l}\text { Postmenopausal } \\
\text { ( } n \text { 175/24 118) }\end{array}$} & \multicolumn{2}{|c|}{$\begin{array}{l}\text { Premenopausal } \\
\quad(n 44 / 3030)\end{array}$} & \multicolumn{2}{|c|}{$\begin{array}{l}\text { Postmenopausal } \\
\text { (n 163/24 115) }\end{array}$} \\
\hline & $\mathrm{HR}$ & $99 \% \mathrm{Cl}$ & $\mathrm{HR}$ & $99 \% \mathrm{Cl}$ & $\mathrm{HR}$ & $99 \% \mathrm{Cl}$ & $\mathrm{HR}$ & $99 \% \mathrm{Cl}$ & $\mathrm{HR}$ & $99 \% \mathrm{Cl}$ & $\mathrm{HR}$ & $99 \% \mathrm{Cl}$ \\
\hline Roots and tubers $/ 66 \mathrm{~g}$ & 0.86 & $0.60,1.22$ & 0.98 & $0.85,1 \cdot 12$ & 0.69 & $0.20,2.38$ & 0.97 & $0.69,1.37$ & 0.64 & $0.26,1.60$ & 1.20 & $0.94,1.53$ \\
\hline Total vegetables $/ 150 \mathrm{~g}$ & 0.94 & $0.79,1 \cdot 13$ & 1.01 & $0.94,1.08$ & $1 \cdot 18$ & $0.71,1.96$ & 0.96 & $0.81,1.14$ & 0.82 & $0.58,1.18$ & 1.09 & $0.95,1.25$ \\
\hline \multicolumn{13}{|l|}{ Fruits } \\
\hline Stone fruits $/ 49 \mathrm{~g}$ & 0.60 & $0.31,1 \cdot 16$ & $1 \cdot 13$ & $0.97,1.33$ & 8.93 & $0.38,207.5$ & $1 \cdot 11$ & $0.72,1.70$ & 0.14 & $0.01,3.50$ & 0.98 & $0.52,1.87$ \\
\hline $\begin{array}{l}\text { Deep orange and yellow } \\
\text { fruits } / 118 \mathrm{~g}\end{array}$ & 0.70 & $0.44,1 \cdot 11$ & $1 \cdot 12$ & $0.93,1.35$ & 0.65 & $0.15,2.90$ & 0.78 & $0.41,1.49$ & 0.09 & $0.01,1.07$ & $1 \cdot 20$ & $0.79,1.81$ \\
\hline Grapes $/ 100 \mathrm{~g}$ & 0.91 & $0.64,1.29$ & 0.95 & $0.80,1.13$ & $1 \cdot 11$ & $0.20,6.05$ & 0.93 & $0.60,1.42$ & 1.08 & $0.21,5.62$ & 1.04 & $0.73,1.49$ \\
\hline Citrus family fruits $/ 92 \mathrm{~g}$ & 1.02 & $0.76,1.37$ & 1.06 & $0.93,1.21$ & 0.89 & $0.16,4.97$ & 0.85 & $0.58,1.25$ & 0.13 & $0.02,0.81$ & 1.06 & $0.76,1.48$ \\
\hline Rhubarb/130 g & 0.80 & $0 \cdot 29,2 \cdot 17$ & 0.93 & $0.64,1.33$ & 0.26 & $0.01,11.2$ & 0.83 & $0.31,2.21$ & 0.47 & $0.06,3.88$ & 1.19 & $0.59,2.38$ \\
\hline Berries $/ 48 \mathrm{~g}$ & 0.87 & $0.68,1.14$ & 1.06 & $0.95,1.18$ & 1.46 & $0.30,7 \cdot 13$ & 0.89 & $0.61,1.29$ & 0.71 & $0.37,1.36$ & 0.88 & $0.59,1.31$ \\
\hline Bananas $/ 100 \mathrm{~g}$ & 0.94 & $0.72,1.24$ & 1.09 & $0.94,1.25$ & 0.65 & $0.24,1.81$ & 0.96 & $0.67,1.39$ & 0.44 & $0.15,1.31$ & 1.32 & $0.97,1.80$ \\
\hline Pomes $/ 116 \mathrm{~g}$ & 0.90 & $0.71,1.14$ & 0.99 & $0.90,1.09$ & 1.25 & $0.60,2 \cdot 61$ & 0.93 & $0.73,1 \cdot 19$ & 0.62 & $0.24,1.61$ & 1.03 & $0.82,1 \cdot 30$ \\
\hline Total fruits $/ 150 \mathrm{~g}$ & 0.94 & $0.84,1.05$ & 1.02 & $0.97,1.07$ & 0.97 & $0.64,1.47$ & 0.93 & $0.80,1.08$ & 0.63 & $0.40,0.99$ & 1.06 & $0.94,1.19$ \\
\hline Dried fruits $/ 28 \mathrm{~g}$ & 1.06 & $0.96,1.16$ & 1.04 & $0.94,1.15$ & 0.99 & $0.25,3.93$ & 0.55 & $0.31,0.98$ & 0.35 & $0.04,2.86$ & $1 \cdot 14$ & $0.99,1.31$ \\
\hline \multicolumn{13}{|l|}{ Other food groups } \\
\hline Sauces/83g & 2.52 & $0.38,16.7$ & 1.30 & $0.66,2.58$ & - & - & 1.91 & $0.40,9 \cdot 12$ & 8.89 & $0.37,215.9$ & 1.28 & $0.22,7.49$ \\
\hline Pickles/chutneys $/ 35 \mathrm{~g}$ & 1.35 & $0.79,2.30$ & 0.85 & $0.60,1.22$ & $2 \cdot 31$ & $0.23,22.9$ & 1.01 & $0.46,2 \cdot 21$ & 2.35 & $0.18,30.5$ & 0.68 & $0.25,1.82$ \\
\hline Soups $/ 163 \mathrm{~g}$ & 0.87 & $0.41,1.83$ & 1.08 & $0.84,1.38$ & - & - & 1.00 & $0.54,1.85$ & 1.01 & $0.32,3.17$ & 1.05 & $0.57,1.91$ \\
\hline $\begin{array}{l}\text { Confectionery and spreads/ } \\
44 \mathrm{~g}\end{array}$ & 0.95 & $0.84,1.08$ & 1.00 & $0.92,1.09$ & 0.89 & $0.51,1.55$ & 0.93 & $0.74,1.17$ & 0.89 & $0.55,1.45$ & 0.99 & $0.80,1.23$ \\
\hline Nuts and seeds $/ 24 \mathrm{~g}$ & 1.03 & $0.90,1 \cdot 18$ & 1.04 & $0.92,1 \cdot 16$ & 1.13 & $0.54,2.36$ & 0.70 & $0.43,1.14$ & 0.39 & $0.10,1.51$ & 1.02 & $0.76,1.38$ \\
\hline Savoury snacks/26g & 0.85 & $0.47,1.52$ & 1.06 & $0.82,1.38$ & 1.64 & $0.25,10 \cdot 8$ & 1.31 & $0 \cdot 75,2 \cdot 27$ & 1.01 & $0 \cdot 19,5 \cdot 34$ & 1.24 & $0.67,2 \cdot 28$ \\
\hline Biscuits/15g & $1 \cdot 17$ & $1.00,1.38$ & 1.00 & $0.93,1.09$ & 0.93 & $0.45,1.93$ & 1.01 & $0.84,1.23$ & 1.40 & $0.75,2.60$ & 0.93 & $0.74,1.18$ \\
\hline Cakes/66g & 0.83 & $0.45,1.52$ & 0.84 & $0.57,1.22$ & 0.06 & $0.00,1.82$ & 0.95 & $0 \cdot 41,2 \cdot 21$ & 0.24 & $0 \cdot 01,5 \cdot 17$ & 1.06 & $0.48,2.37$ \\
\hline Pastries and puddings $/ 84 \mathrm{~g}$ & 1.47 & $0.98,2.19$ & 1.08 & $0.83,1.39$ & 0.26 & $0.01,4.75$ & $1 \cdot 16$ & $0 \cdot 64,2 \cdot 11$ & $1 \cdot 12$ & $0.06,22.0$ & 0.76 & $0.35,1.61$ \\
\hline \multicolumn{13}{|l|}{ Drinks and beverages } \\
\hline $\mathrm{Tea} / 260 \mathrm{~g}$ & 0.98 & $0.90,1.06$ & 0.99 & $0.95,1.03$ & $1 \cdot 14$ & $0.84,1.55$ & 1.02 & $0.92,1.13$ & 0.98 & $0.77,1.24$ & 0.94 & $0.84,1.04$ \\
\hline Herbal tea/260 g & 1.06 & $0.87,1.29$ & 1.00 & $0.91,1.09$ & 1.49 & $0.71,3.11$ & 0.89 & $0.68,1 \cdot 16$ & 0.76 & $0.34,1.72$ & 0.96 & $0.74,1.25$ \\
\hline Coffee/190 g & 1.03 & $0.95,1.11$ & 1.01 & $0.97,1.06$ & 1.03 & $0.76,1.39$ & 1.01 & $0.91,1.13$ & $1 \cdot 16$ & $0.87,1.54$ & 1.07 & $0.96,1.19$ \\
\hline Other hot beverages $/ 23 \mathrm{~g}$ & 1.02 & $0.79,1.31$ & 1.01 & $0.89,1 \cdot 15$ & 0.25 & $0.04,1.47$ & 1.05 & $0.79,1.42$ & $1 \cdot 18$ & $0.54,2.58$ & 1.08 & $0.80,1.46$ \\
\hline Juices/145g & 0.89 & $0.72,1.10$ & 0.99 & $0.90,1.09$ & 1.09 & $0.51,2.33$ & 0.96 & $0.76,1 \cdot 23$ & 0.65 & $0.31,1.35$ & 1.02 & $0.81,1.29$ \\
\hline Soft drinks/111g & 1.04 & $0.87,1.23$ & 1.03 & $0.90,1.19$ & 0.98 & $0.36,2.67$ & $1 \cdot 15$ & $0.88,1.50$ & 1.52 & $0.80,2.88$ & 1.09 & $0.80,1.48$ \\
\hline $\begin{array}{l}\text { Low-energy/diet soft drinks/ } \\
161 \mathrm{~g}\end{array}$ & 1.00 & $0.78,1 \cdot 29$ & 1.03 & $0.90,1 \cdot 18$ & 0.34 & $0 \cdot 05,2 \cdot 18$ & $1 \cdot 27$ & $1.00,1.61$ & 1.70 & $0.64,4.50$ & 1.01 & $0.70,1.45$ \\
\hline Wines/glass $\ddagger$ & 0.98 & $0.81,1.18$ & 1.03 & $0.92,1.15$ & 1.24 & $0.40,3.79$ & 0.85 & $0.61,1 \cdot 18$ & 0.89 & $0.50,1.59$ & 1.01 & $0.75,1.36$ \\
\hline Beer and cider/half pint $\ddagger$ & 1.09 & $0.65,1.83$ & $1 \cdot 15$ & $0.94,1.42$ & $4 \cdot 11$ & $0.44,38.4$ & 1.26 & $0.81,1.97$ & 1.81 & $0.93,3.53$ & 1.05 & $0.56,1.97$ \\
\hline Port, sherry, liqueurs/glass $\ddagger$ & 1.23 & $0.59,2.60$ & 1.01 & $0.73,1.39$ & - & - & 0.95 & $0.42,2.15$ & 0.58 & $0 \cdot 16,2 \cdot 14$ & 1.31 & $0.77,2 \cdot 21$ \\
\hline Spirits/measureł & 1.07 & $0.80,1.43$ & 1.05 & $0.87,1.28$ & 0.76 & $0.01,76 \cdot 3$ & 0.49 & $0 \cdot 20,1 \cdot 21$ & $1 \cdot 21$ & $0.51,2.86$ & $1 \cdot 12$ & $0.74,1.71$ \\
\hline
\end{tabular}

* Fully adjusted for age, ethanol intake, duration of breast-feeding, physical activity, smoking, social class and menopausal status.

† Fully adjusted for age, ethanol intake, duration of breast-feeding, physical activity, smoking, social class, menopausal status, history of diabetes and history of hypertension.

t Not adjusted for ethanol intake. 
dietary lignans. Lignans, a type of phyto-oestrogens are plant compounds having structural similarity to 17 -oestrodiol. They can lower endogenous oestrogen levels by potentially binding to oestrogen receptors ${ }^{(59)}$, hence reducing the risk of endometrial cancer.

Contrary to a previous case-control study undertaken in Chinese women, which demonstrated an inverse association between white button mushrooms and risk of ovarian cancer ${ }^{(60)}$, our findings showed weak evidence of an increased risk in relation to the consumption of a standard portion of mushrooms per d. Furthermore according to a study among Korean women, high mushroom intake was reportedly associated with a lower risk of breast cancer among premenopausal women and a stronger association was reported among premenopausal women with oestrogen-receptorpositive and progesterone-receptor-positive tumours ${ }^{(61)}$. However, in this study we do not have this level of detail in terms of types of mushroom consumption and breast cancer by hormone receptor type. This difference could also be attributed to the fact that Chinese cohorts most commonly consume fresh mushrooms, while in Europe the use of canned mushrooms is more widespread. In addition, in the UK, there is no other evidence suggesting that mushrooms can increase or decrease the risk of cancer ${ }^{(62)}$.

Strengths of this study include the prospective study design, a long follow-up time and large sample size. This is also the first study in the UK looking at multiple food groups in relation to the risk of breast, endometrial and ovarian cancers. We were also able to study the associations with specific types of meat, cereal products (wholegrain or refined) and dairy products (high fat or low fat). We adjusted for a wide range of confounders including sociodemographic and lifestyle using a consistent method (directed acyclic graph). However, as in any observational study, residual confounding is still possible. A limitation of our study was the inability to determine whether the associations varied according to the hormone receptor status of tumours, due to the lack of these data at present in this cohort. The UKWCS will soon be expanding to include additional details on the tumour types. Moreover, the use of an FFQ for dietary assessment could also be prone to low accuracy due to recall bias. However, the FFQ is a useful tool in providing a snapshot of the dietary habit over a longer period of time. Regression dilution might also be an issue, given participants' diets may have changed over time, potentially introducing further measurement error. This study also does not take into account the use of pesticides which is also a potential carcinogen influencing cancer risk in women. Our sample was also more health conscious, given the high number of vegetarians in our sample population and more well-off participants than the general population. However, our study still included women from a range of different backgrounds, which implies that findings of this study may be extrapolated to other countries.

Primary prevention of cancer is important and a matter of consideration in public health. While factors such as parity, age at onset of natural menopause and family history are well established to have a link with the risk of breast, endometrial and ovarian cancer, they are non-modifiable risk factors. However, diet which has been shown to either increase or decrease the risk of carcinogenesis makes focus on diet an interesting opportunity in cancer prevention.

To summarise, this study suggests a link between specific foods: processed meat, total meat, tomatoes, dried fruits and wholegrain products and the risk of breast as well as endometrial cancer while a relationship between diet and risk of ovarian cancer is less evident. These findings support the call for further randomised controlled trials of dietary interventions to reduce the risk of these hormone-related cancers among preand postmenopausal women.

\section{Acknowledgements}

The authors thank the UKWCS steering group who initiated the study, collected data, managed and processed information of the cohort as well as the participants of the UKWCS.

Y. D. is in receipt of a scholarship from the Commonwealth Scholarships Commission, UK. J. E. C. acknowledges additional support from the Medical Research Council (MR/L02019X/1). The Commonwealth Scholarships Commission and the Medical Research Council had no role in design and conduct of the study; collection, management, analysis, and interpretation of the data; preparation, review, and approval of the manuscript; or the decision to submit the manuscript for publication.

J. E. C. initiated and developed the cohort. Y. D. was primarily responsible for data analysis and writing the manuscript. All authors were involved in the study design, interpretation of findings, editing and approving the article.

J. E. C. is a director of the University of Leeds spin out company Dietary Assessment Ltd.

\section{Supplementary material}

For supplementary material/s referred to in this article, please visit https://doi.org/10.1017/S0007114518003665

\section{References}

1. Office for National Statistics (2018) Statistical bulletin: cancer registration statistics, England: 2016. https://www.ons.gov.uk/ peoplepopulationandcommunity/healthandsocialcare/con ditionsanddiseases/bulletins/cancerregistrationstatisticsengland/ final2016 (accessed May 2018).

2. Cancer Research UK. Cancer statistics by type. http://www. cancerresearchuk.org/health-professional/cancer-statistics/statistics-by-cancer-type (accessed May 2018).

3. Kelsey JL, Gammon MD \& John EM (1993) Reproductive factors and breast cancer. Epidemiol Rev 15, 36-47.

4. McPherson CP, Sellers TA, Potter JD, et al. (1996) Reproductive factors and risk of endometrial cancer: the Iowa Women's Health study. Am J Epidemiol 143, 1195-1202.

5. Hunn J \& Rodriguez GC (2012) Ovarian cancer: etiology, risk factors, and epidemiology. Clin Obstet Gynecol 55, 3-23.

6. Cramer DW (2012) The epidemiology of endometrial and ovarian cancer. Hematol Oncol Clin North Am 26, 1-12.

7. Jones ME, Schoemaker MJ, Wright LB, et al. (2017) Smoking and risk of breast cancer in the Generations Study cohort. Breast Cancer Res 19, 118.

8. Gallagher EJ \& LeRoith D (2013) Epidemiology and molecular mechanisms tying obesity, diabetes, and the 
metabolic syndrome with cancer. Diabetes care 36, Suppl. 2, S233-S239.

9. de Oliveira EP, McLellan KCP, Vaz de Arruda Silveira L, et al (2012) Dietary factors associated with metabolic syndrome in Brazilian adults. Nutr J 11, 13.

10. Henderson BE \& Feigelson HS (2000) Hormonal carcinogenesis. Carcinogenesis 21, 427-433.

11. Thomas HV, Davey GK \& Key TJ (1999) Oestradiol and sex hormone-binding globulin in premenopausal and postmenopausal meat-eaters, vegetarians and vegans. Br J Cancer 80, $1470-1475$.

12. Barnard ND, Scialli AR, Hurlock D, et al. (2000) Diet and sexhormone binding globulin, dysmenorrhea, and premenstrual symptoms. Obstet Gynecol 95, 245-250.

13. Gann PH, Chatterton RT, Gapstur SM, et al. (2003) The effects of a low-fat/high-fiber diet on sex hormone levels and menstrual cycling in premenopausal women: a 12-month randomized trial (the diet and hormone study). Cancer $\mathbf{9 8}$, 1870-1879.

14. Boyd NF, Lockwood GA, Greenberg CV, et al. (1997) Effects of a low-fat high-carbohydrate diet on plasma sex hormones in premenopausal women: results from a randomized controlled trial. Canadian Diet and Breast Cancer Prevention Study Group. Br J Cancer 76, 127-135.

15. World Cancer Research Fund/American Institute for Cancer Research (2018) Diet, nutrition, physical activity and cancer: a global perspective. Continuous update project expert report 2018. http://www.dietandcancerreport.org (accessed April 2018).

16. Samavat H \& Kurzer MS (2015) Estrogen metabolism and breast cancer. Cancer Lett 356, 231-243.

17. Allen NE, Key TJ, Dossus L, et al. (2008) Endogenous sex hormones and endometrial cancer risk in women in the European Prospective Investigation into Cancer and Nutrition (EPIC). Endocr Relat Cancer 15, 485-497.

18. Lukanova A \& Kaaks R (2005) Endogenous hormones and ovarian cancer: epidemiology and current hypotheses. Cancer Epidemiol Biomarkers Prev 14, 98-107.

19. Cade JE, Burley VJ, Alwan NA, et al. (2017) Cohort profile: the UK Women's Cohort Study (UKWCS). Int I Epidemiol 46, e11.

20. Taylor EF, Burley VJ, Greenwood DC, et al. (2007) Meat consumption and risk of breast cancer in the UK Women's Cohort Study. Br J Cancer 96, 1139-1146.

21. World Cancer Research Fund/American Institute for Cancer Research (2018) Changes since the 2007 second expert report: important shifts in emphasis. https://www.wcrf.org/dietand cancer/changes-since-2007-second-expert-report (accessed June 2018).

22. Dunneram Y, Greenwood DC, Burley VJ, et al. (2018) Dietary intake and age at natural menopause: results from the UK Women's Cohort Study. J Epidemiol Community Health $\mathbf{7 2}$, 733-740.

23. Food Standards Agency (2002) Food Portion Sizes. London: The Stationery Office.

24. Cade JE, Taylor EF, Burley VJ, et al. (2011) Does the Mediterranean dietary pattern or the healthy diet index influence the risk of breast cancer in a large British cohort of women? Eur J Clin Nutr 65, 920-928.

25. Clague J \& Bernstein L (2012) Physical activity and cancer. Curr Oncol Rep 14, 550-558.

26. Bagnardi V, Rota M, Botteri E, et al. (2015) Alcohol consumption and site-specific cancer risk: a comprehensive dose-response meta-analysis. Br J Cancer 112, 580-593.

27. Stockwell HG \& Lyman GH (1987) Cigarette smoking and the risk of female reproductive cancer. Am J Obstet Gynecol 157, $35-40$.
28. Wang L, Li J \& Shi Z (2015) Association between breastfeeding and endometrial cancer risk: evidence from a systematic review and meta-analysis. Nutrients 7, 5697-5711.

29. Zhan B, Liu X, Li F, et al. (2015) Breastfeeding and the incidence of endometrial cancer: a meta-analysis. Oncotarget $\mathbf{6}$, 38398-38409.

30. Su D, Pasalich M, Lee AH, et al. (2013) Ovarian cancer risk is reduced by prolonged lactation: a case-control study in southern China. Am J Clin Nutr 97, 354-359.

31. Liu L, Deapen D \& Bernstein L (1998) Socioeconomic status and cancers of the female breast and reproductive organs: a comparison across racial/ethnic populations in Los Angeles County, California (United States). Cancer Causes Control 9 , 369-380.

32. Friberg E, Mantzoros CS \& Wolk A (2007) Diabetes and risk of endometrial cancer: a population-based prospective cohort study. Cancer Epidemiol Biomarkers Prev 16, 276-280.

33. Aune D, Sen A \& Vatten LJ (2017) Hypertension and the risk of endometrial cancer: a systematic review and meta-analysis of case-control and cohort studies. Sci Rep 7, 44808.

34. Persson I (2000) Estrogens in the causation of breast, endometrial and ovarian cancers - evidence and hypotheses from epidemiological findings. J Steroid Biochem Mol Biol 74, 357-364.

35. Simin J, Tamimi R, Lagergren J, et al. (2017) Menopausal hormone therapy and cancer risk: an overestimated risk? Eur $J$ Cancer 84, 60-68.

36. Beral V (2003) Breast cancer and hormone-replacement therapy in the Million Women Study. Lancet 362, 419-427.

37. Salazar-Martinez E, Lazcano-Ponce EC, Lira-Lira GG, et al. (1999) Reproductive factors of ovarian and endometrial cancer risk in a high fertility population in Mexico. Cancer Res 59, 3658-3662.

38. Tsilidis KK, Allen NE, Key TJ, et al. (2011) Oral contraceptive use and reproductive factors and risk of ovarian cancer in the European Prospective Investigation into Cancer and Nutrition. Br J Cancer 105, 1436-1442.

39. Anderson JJ, Darwis NDM, Mackay DF, et al. (2018) Red and processed meat consumption and breast cancer: UK Biobank cohort study and meta-analysis. Eur J Cancer 90, 73-82.

40. Pala V, Krogh V, Berrino F, et al. (2009) Meat, eggs, dairy products, and risk of breast cancer in the European Prospective Investigation into Cancer and Nutrition (EPIC) cohort. Am J Clin Nutr 90, 602-612.

41. Fiolet T, Srour B, Sellem L, et al. (2018) Consumption of ultraprocessed foods and cancer risk: results from NutriNet-Santé prospective cohort. BMJ 360, k322.

42. Pouchieu C, Deschasaux M, Hercberg S, et al. (2014) Prospective association between red and processed meat intakes and breast cancer risk: modulation by an antioxidant supplementation in the SU.VI.MAX randomized controlled trial. Int J Epidemiol 43, 1583-1592.

43. Fabio L, Carlo LV, Silvia F, et al. (1993) Dietary factors and the risk of endometrial cancer. Cancer 71, 3575-3581.

44. Arem H, Gunter MJ, Cross AJ, et al. (2013) A prospective investigation of fish, meat and cooking-related carcinogens with endometrial cancer incidence. BrJ Cancer 109, 756-760.

45. Cross AJ, Leitzmann MF, Gail MH, et al. (2007) A prospective study of red and processed meat intake in relation to cancer risk. PLoS Med 4, e325.

46. Inoue-Choi M, Sinha R, Gierach GL, et al. (2016) Red and processed meat, nitrite, and heme iron intakes and postmenopausal breast cancer risk in the NIH-AARP Diet and Health Study. Int J Cancer 138, 1609-1618.

47. Zheng W, Gustafson DR, Moore D, et al. (1998) Well-done meat intake and the risk of breast cancer. J Natl Cancer Inst 90, 1724-1729. 
48. Nagao M, Ushijima T, Wakabayashi K, et al. (1994) Dietary carcinogens and mammary carcinogenesis. Induction of rat mammary carcinomas by administration of heterocyclic amines in cooked foods. Cancer 74, 1063-1069.

49. Carlson MJ, Thiel KW, Yang S, et al. (2012) Catch it before it kills: progesterone, obesity, and the prevention of endometrial cancer. Discov Med 14, 215-222.

50. Bremer AA \& Miller WL (2014) Chapter 13 - Regulation of steroidogenesis A2 - Ulloa-Aguirre, Alfredo. In Cellular Endocrinology in Health and Disease, pp. 207-227 [PM Conn, editor]. Boston, MA: Academic Press.

51. Eliassen AH, Hendrickson SJ, Brinton LA, et al. (2012) Circulating carotenoids and risk of breast cancer: pooled analysis of eight prospective studies. J Natl Cancer Inst 104, 1905-1916.

52. Levy J, Bosin E, Feldman B, et al. (1995) Lycopene is a more potent inhibitor of human cancer cell proliferation than either alpha-carotene or beta-carotene. Nutr Cancer 24, 257-266.

53. Karas M, Amir H, Fishman D, et al. (2000) Lycopene interferes with cell cycle progression and insulin-like growth factor I signaling in mammary cancer cells. Nutr Cancer 36, 101-111.

54. Lutz M, Hernández J \& Henríquez C (2015) Phenolic content and antioxidant capacity in fresh and dry fruits and vegetables grown in Chile. CyTA - J Food 13, 541-547.
55. Capanoglu E (2014) Investigating the antioxidant potential of Turkish dried fruits. Int J Food Prop 17, 690-702.

56. Goodman MT, Wilkens LR, Hankin JH, et al. (1997) Association of soy and fiber consumption with the risk of endometrial cancer. Am J Epidemiol 146, 294-306.

57. Bidoli E, Pelucchi C, Zucchetto A, et al. (2010) Fiber intake and endometrial cancer risk. Acta Oncol 49, 441-446.

58. Sowers MR, Crawford S, McConnell DS, et al. (2006) Selected diet and lifestyle factors are associated with estrogen metabolites in a multiracial/ethnic population of women. $J$ Nutr 136, 1588-1595.

59. Kuiper GGJM, Lemmen JG, Carlsson B, et al. (1998) Interaction of estrogenic chemicals and phytoestrogens with estrogen receptor $\beta$. Endocrinology 139, 4252-4263.

60. Lee AH, Pasalich M, Su D, et al. (2013) Mushroom intake and risk of epithelial ovarian cancer in southern Chinese women. Int J Gynecol Cancer 23, 1400-1405.

61. Shin A, Kim J, Lim SY, et al. (2010) Dietary mushroom intake and the risk of breast cancer based on hormone receptor status. Nutr Cancer 62, 476-483.

62. Cancer Research UK (2015) Mushrooms in cancer treatment. http://www.cancerresearchuk.org/about-cancer/cancer-ingeneral/treatment/complementary-alternative-therapies/individualtherapies/mushrooms-in-cancer-treatment (accessed June 2018).

4

